\title{
TANNINS IN SOME AGRO FORESTRY SPECIES OF LOCAL INTEREST
}

\section{Y J D JW Seresinhe, Thakshala Seresinhe, and K K. Pathirana Faculty of Agriculture, University of Ruhuna}

Total and condensed tannin contents were determined in six agro forestry species used by local farmers to feed ruminants. Total and condensed tannins were determined by Folin-Ciocalteu and Butanol-Hcl methods respectively. Total tannin (TT) content ranged from $5.41 \%$ for Calliandra calothyrsus to $0.15 \%$ for Erythrina indica. Condensed tannins (CT) contents ranged from $6.92 \%$ in Acacia auriculiformis to $0.78 \%$ in Gliricidia sepium. The percentages of CT were $6.40 \%, 4.60 \%$, and $1.58 \%$ respectively for Artocarpus heterophyllus, C. calothyrsus, and Leucaena leucocephala. Among the six species tested, $E$. indica, was free of CT while having traces of TT. On the basis of their respective CT contents $G$. sepium was classified as containing traces of $\mathrm{CT}(<1 \%)$, while $L$. leucocephala classified as containing low level of CT (1-3\%). C. calothyrsus was classified as containing medium level of CT (4-6\%) while $A$. auriculiformis and $A$. heterophyllus classified as containing high level of CT $(>6 \%)$.

In a further study, it was found that tender leaves and stems $(0-30 \mathrm{~cm}$ from the tip) of $C$. calothyrsus, and A. auriculiformis contained higher levels of TT and CT as compared with mature leaves and stems $(30-60 \mathrm{~cm}$ from the tip) of the same species. In contrast tender leaves and stems of A. heterophyllus contained low levels TT as compared with mature leaves and stems.

The results suggest that TT and CT contents of tested six species are different due to their inherent characteristics. It was also evident that tannins are present not only in leguminous species but also in non-leguminous species such as $A$. heterophyllus. The increase in TT and CT contents in tender parts of $C$. callothyrsus and A. auriculiformis may be associated with the chemical defence mechanisms found in younger plant parts. Further investigations are necessary to verify th: effects of tannins on their nutritive value of above forages.

Proceedings of the Ninth Annual Forestry and Environment Symposium 2003 of the Department of Forestry and Environmental Science, University of Sri Jayewardenepura, Sri Lanka 